

\title{
Geometry-induced magnetoelectric effect enhancement and noise floor reduction in Metglas/piezofiber sensors
}

\author{
Yaojin Wang, ${ }^{\text {a) }}$ Menghui Li, D. Hasanyan, Junqi Gao, Jiefang Li, and D. Viehland \\ Materials Science and Engineering, Virginia Tech, Blacksburg, Virginia 24061, USA
}

(Received 2 May 2012; accepted 5 July 2012; published online 29 August 2012)

The geometry-dependent magnetoelectric (ME) effect was theoretically and experimentally investigated for multi-push-pull mode $\operatorname{Metglas} / \mathrm{Pb}(\mathrm{Zr}, \mathrm{Ti}) \mathrm{O}_{3}$ sandwich-like laminates. Such structures hold promise for passive sensor applications. A geometry-induced significant enhancement in the ME coefficient and an effective reduction in the equivalent magnetic noise was observed due to an increase in the Metglas width fraction. (c) 2012 American Institute of Physics. [http://dx.doi.org/10.1063/1.4737906]

Multiferroic materials, which display simultaneous magnetic and electric ordering, have recently stimulated much scientific and technological interest. ${ }^{1}$ In particular, the magnetoelectric (ME) effect - a change of electrical polarization $P$ (or voltage $V$ ) due to an applied magnetic field $H$ or conversely a change of magnetization $M$ (or induction $B$ ) due to an applied electric field $E$ - has been studied in multiferroics. ${ }^{2}$ The ME effect has been observed as an intrinsic effect in single phase materials. However, a technological viable inherent ME coupling has not yet been found in such compounds. ${ }^{3}$ The coexistence of ferromagnetic and ferroelectric subsystems engenders composites with a product (extrinsic) ME effect: the composite exhibits a response that is not available in the individual component phases. Thus, an additional degree of freedom in the design of passive magnetic sensors is enabled, offering a potential alternative to Hall and fluxgate sensors that require electric current. With regards to sensor applications, the product $\mathrm{ME}$ effect of composites has been widely investigated in recent years ranging from laminated layers to nano-structured thin films. ${ }^{1,4,5}$ A milestone in the development of ME composites was the appearance of the multi-push-pull mode, consisting of magnetostrictive Metglas alloys and piezofibers with interdigitated-electrodes. ${ }^{6}$

However, the quest for materials exhibiting large ME coupling remains a major challenge. The solution of which could unlock technological advances in numerous fields. For instance, materials with strong ME effects could lead to high magnetic field sensitivities and low equivalent magnetic noises in passive magnetic sensors. Although, the geometrydependent ME coefficient has been extensively investigated by considering the thickness fraction of piezoelectric or magnetostrictive phases, comprehensive studies of the geometrydependent ME coefficient was notably deficient. Here, we theoretically and experimentally demonstrate a geometryinduced ME enhancement through strain engineering. A theoretical model is presented that predicts the ME effect can be significantly enhanced when the width fraction of Metglas is increased. Experimental results then reveal good agreement with predicted ones.

We consider a coordinate system for a multi-pull-pull mode Metglas/piezofiber heterostructure in the $(2,3)$ plane.

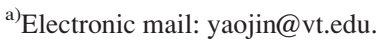

By assuming that the polarization of the piezofiber can be uniformly arranged along the longitudinal direction (3-axis), the representation for the multi-push-pull configuration can then be simplified to a multi-L-L mode. An averaging method was used for deriving the effective material parameters of the laminate. The piezoelectric constitutive equations can be written as

$$
{ }^{p} S_{i}={ }^{p} S_{i j}^{p} T_{j}+{ }^{p} d_{k i}^{p} E_{k}, \quad{ }^{p} D_{k}={ }^{p} d_{k i}^{p} T_{i}+{ }^{p} \varepsilon_{k n}{ }^{p} E_{n},
$$

where ${ }^{P} S_{i}$ and ${ }^{p} T_{j}$ are the strain and stress tensor components of the piezoelectric phase; ${ }^{p} E_{k},{ }^{p} E_{n}$, and ${ }^{p} D_{k}$ are the vector components of the electric field and electric displacement; ${ }^{p} s_{i j}$ and ${ }^{p} d_{k i}$ are the compliance and piezoelectric coefficients; and ${ }^{p} \varepsilon_{k n}$ is the permittivity tensor matrix of the piezoelectric phase. The magnetostrictive phase can be described by the following constitutive equations:

$$
{ }^{m} S_{i}={ }^{m} s_{i j}{ }^{m} T_{j}+{ }^{m} q_{k i}{ }^{m} H_{k},{ }^{m} B_{k}={ }^{m} q_{k i}{ }^{m} T_{i}+{ }^{m} \mu_{k n}{ }^{m} H n,
$$

where ${ }^{m} S_{i}$ and ${ }^{m} T_{j}$ are the strain and stress tensor components of the magnetostrictive phase; ${ }^{m} H_{k},{ }^{m} H_{n}$, and ${ }^{m} B_{k}$ are the vector components of the magnetic field and magnetic flux induction; ${ }^{m} s_{i j}$ and ${ }^{m} q_{k i}$ are the compliance and piezomagnetic coefficients; and ${ }^{m} \mu_{k n}$ is the permeability matrix of the magnetostrictive phase.

Our analysis assumed small deformations, linear material properties, perfect interfacial bonding, non-shear-lag effect (i.e., rigid components), ${ }^{12}$ and open-circuit conditions. Furthermore, we only considered the geometry effect in the width and thickness directions. For the solutions of Eqs. (1) and (2), the following boundary conditions of a traction free plane stress in the cross-sectional directions (i.e., equivalence of inplane strains and equilibrium) were used, yielding

$$
\begin{aligned}
& { }^{m} S_{i}={ }^{p} S_{i}(i=1,3), \quad{ }^{m} T_{2}={ }^{p} T_{2}=0, \\
& (1-\nu)(1-\omega)^{m} T_{3}+v \omega \omega^{p} T_{3}=0, \\
& (1-\nu)^{m} T_{1}+v^{p} T_{1}=0, \quad{ }^{p} D_{3}=0,
\end{aligned}
$$

where $v=t_{p} /\left(t_{p}+2 t_{m}\right)$ and $\omega=w_{p} /\left(w_{p}+w_{m}\right) ; w_{\mathrm{m}}$ and $t_{\mathrm{m}}$ denote the width and thickness of magnetostrictive; and $w_{\mathrm{p}}$ and $t_{\mathrm{p}}$ denote the width and thickness of piezoelectric layer, as illustrated in Figure 1(c).

From Eqs. (1), (2), and (3), we can obtain 


$$
\begin{aligned}
{\left[{ }^{m} s_{11}+\frac{{ }^{p} s_{11}(1-v)}{v}\right]{ }^{m} T_{2}+} & {\left[{ }^{m} s_{13}+\frac{{ }^{p} s_{13}(1-v)(1-\omega)}{v \omega}\right]{ }^{m} T_{3}-{ }^{p} d_{31}{ }^{p} E_{3}=-{ }^{m} q_{33}{ }^{m} H_{3}, } \\
{\left[{ }^{m} s_{13}+\frac{{ }^{p} s_{13}(1-v)}{v}\right]{ }^{m} T_{2}+} & {\left[{ }^{m} s_{11}+\frac{{ }^{p} s_{33}(1-v)(1-\omega)}{v \omega}\right]{ }^{m} T_{3}-{ }^{p} d_{31}{ }^{p} E_{3}=-{ }^{m} q_{31}{ }^{m} H_{3}, } \\
& \frac{{ }^{p} d_{31}(1-v)}{v}{ }^{m} T_{2}+\frac{{ }^{p} d_{33}(1-v)(1-\omega)}{v \omega}{ }^{m} T_{3}-{ }^{p} \varepsilon_{33}{ }^{p} E_{3}=0 .
\end{aligned}
$$

Next, we define the effective compliance coefficients as

$$
A={ }^{m} s_{11}+\frac{{ }^{p} s_{11}(1-v)}{v} ; \quad B={ }^{m} s_{13}+\frac{{ }^{p} s_{13}(1-v)(1-\omega)}{v \omega} ; \quad C={ }^{m} s_{13}+\frac{{ }^{p} s_{13}(1-v)}{v} ; \quad D={ }^{m} s_{11}+\frac{{ }^{p} S_{33}(1-v)(1-\omega)}{v \omega} ;
$$

and the effective piezoelectric coefficient as

$$
X=\frac{{ }^{p} d_{31}(1-v)}{v} ; \quad Y=\frac{{ }^{p} d_{33}(1-v)(1-\omega)}{v \omega} .
$$

From Eq. (4) and the above definitions, the ME coefficient in terms of the electric field $\left(E_{3}=v^{p} E_{3}\right)^{7}$ response to an applied magnetic field $\left(\mathrm{H}_{3}\right)$ can be expressed as

$$
\begin{aligned}
\alpha_{E}= & -\left({ }^{m} q_{33} v\right) \times \frac{(Y C-X D)}{-B X^{p} d_{31}+A Y^{p} d_{31}+B C^{p} \varepsilon_{33}-Y C^{p} d_{31}-A D^{p} \varepsilon_{33}+X D^{p} d_{31}} \\
& -\left({ }^{m} q_{31} v\right) \times \frac{B X-A Y}{-B X^{p} d_{31}+A Y^{p} d_{31}+B C^{p} \varepsilon_{33}-Y C^{p} d_{31}-A D^{p} \varepsilon_{33}+X D^{p} d_{31}} .
\end{aligned}
$$

From Eq. (5), the value of $\alpha_{\mathrm{E}}$ can be predicted as a function of piezoelectric phase thickness fraction $\nu$ and width fraction $\omega$ for a Metglas/piezofiber laminate composite using appropriate material parameters, ${ }^{8}$ as shown in Fig. 2. Obviously, a ME coupling was absent in the individual phase $(v=0$, Metglas; $v=1$, PZT). For various width fractions $\omega$, as $v$ was

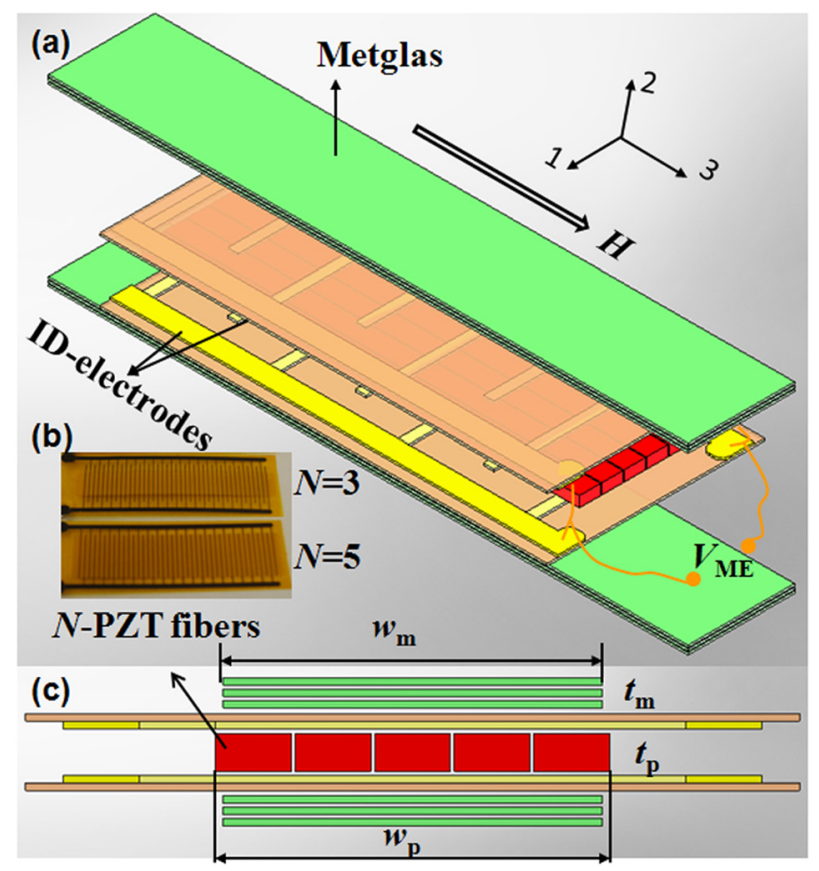

FIG. 1. (a) Three-dimensional and (c) cross-sectional schematic illustration of the multi-push-pull mode Metglas/piezofiber heterostructure. The width of the Metglas layer is $w_{\mathrm{m}}$, and the width of PZT bundle is $w_{\mathrm{p}}$ (i.e., $w_{\mathrm{p}}=2 \mathrm{~N}$, width of each fiber is $2 \mathrm{~mm}$ and $N$ is the number of fibers). (b) Photograph for the PZT-fiber core composites with $N=3$ and 5 . increased from $0<v<1$, the value of $\alpha_{\mathrm{E}}$ increased, reached a maximum value of $\alpha_{\mathrm{E}, \max }$ at $v_{\max }$ (around 0.55 ) and then decreased. It can be seen that the predicted values of $\alpha_{\mathrm{E} \text {,max }}$ were significantly enhanced as the width fraction $\omega$ decreased and $v_{\max }$ slightly shifted to Metglas-rich phase fractions: the values of $\alpha_{\mathrm{E}}$ for $\omega=0.357$ and 0.375 were 42.6 and $40.5 \mathrm{~V} /(\mathrm{cm} \times \mathrm{Oe})$, increasing by a factor of $1.4 \times$ and $1.3 \times$ relative to that of $\alpha_{\mathrm{E}}=31.2$ for $\omega=0.5$, respectively.

To confirm these predictions, three Metglas/PZT-fiber multi-push-pull mode laminates were fabricated. They either had the same width of Metglas $\left(w_{\mathrm{m}}=10 \mathrm{~mm}\right)$ with different widths of the piezofiber layer (i.e., $N=3$ and 5) or the same width of piezofiber layer (i.e., $N=5$ ) with different width of Metglas $\left(w_{\mathrm{m}}=10\right.$ and $\left.18 \mathrm{~mm}\right)$. PZT bundles of dimensions

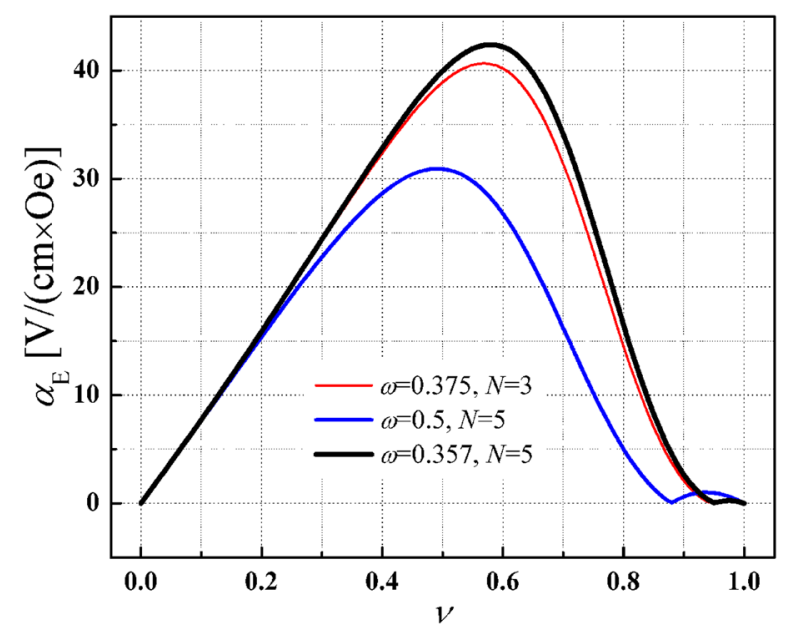

FIG. 2. Predicted ME coefficient $\alpha_{\mathrm{E}}$ as a function of piezofiber thickness fraction $v$ for various piezofiber width fraction $\omega$. 
$40 \mathrm{~mm} \times 6 \mathrm{~mm}$ and $40 \mathrm{~mm} \times 10 \mathrm{~mm}$ served as the cores of the laminates, which consisted of $N=3$ and $N=5$ PZT fiber each with dimensions of $40 \mathrm{~mm} \times 2 \mathrm{~mm} \times 0.18 \mathrm{~mm}$. The PZT fibers were purchased from Smart Materials Corp. (Sarasota, FL) and were oriented along the length direction of the laminates, as shown in Fig. 1(b). Three pairs of Kapton interdigitated copper electrodes with $50 \mu \mathrm{m}$ wide digited spacing at $1 \mathrm{~mm}$ center-to-center were bonded to the top and bottom surfaces of the PZT bundle with epoxy resin. Metglas of composition $\mathrm{Fe}_{74.4} \mathrm{Co}_{21.6} \mathrm{Si}_{0.5} \mathrm{~B}_{3.3} \mathrm{Mn}_{0.1} \mathrm{C}_{0.1}$ were purchased from Vacuumscheltze $\mathrm{GmbH} \& \mathrm{Co}$. (KG, Germany) as a roll which had a layer thickness of $25 \mu \mathrm{m}$ and a layer width of $18 \mathrm{~mm}$. The Metglas samples were cut into foils of surface area of $80 \mathrm{~mm} \times 8 \mathrm{~mm}$ and $80 \mathrm{~mm} \times 18 \mathrm{~mm}$. Three such Metglas layers were then stacked one on top of each other and bonded with epoxy resin (West system 206, USA) using a vacuum bag pressure method. Then, the Metglas layers were symmetrically stacked and bonded to top and bottom sides of the core composites (i.e., $\nu=0.55$ ), as shown in Fig. 1(a).

First, the voltage induced across the ID-electrodes of the three Metglas/PZT-fiber heterostructures were measured with a lock-in amplifier (Stanford SR-850) as a function of dc magnetic bias $\left(H_{\mathrm{dc}}\right)$ and in response to a Helmholtz coil driven by an ac magnetic field of $H_{\mathrm{ac}}=0.1 \mathrm{Oe}$ at a frequency $f=1 \mathrm{kHz}$, as shown in Fig. 3. Both dc and ac fields were applied along the length of the heterostructures. Figure 3 shows the ME coefficient $\alpha_{\mathrm{E}}$ as a function of $H_{\mathrm{dc}}$ for different width fraction of PZT-fiber with $\omega=0.375$ and $N=3$, $\omega=0.5$ and $N=5$, and $\omega=0.357$ and $N=5$. From this figure, it can be seen that the values of $\alpha_{\mathrm{E}}$ increased from nearly zero at $H_{\mathrm{dc}}=0$ Oe to a maximum at a particular $H_{\mathrm{dc}}$, and then subsequently decreased as $H_{\mathrm{dc}}$ was further increased. One can also see that the maximum value of $\alpha_{\mathrm{E}}$ for $\omega=0.375$ and 0.357 were larger than that for $\omega=0.5$, resulting corresponding 1.3 and 1.5 times enhancements in $\alpha_{\mathrm{E}}$. It should be noted that prior reports have focused on $\omega=0.5^{6,9-11}$ Experimentally, $\alpha_{\mathrm{E}}$ for the three laminates exhibited similar trends to the predicted ones. However, due to a nonideal interfacial bonding between magnetostrictive and piezofiber layers, ${ }^{7}$ the three experimental values were $77 \%, 69 \%$, and $67 \%$ of the theoretical predicted ones. This

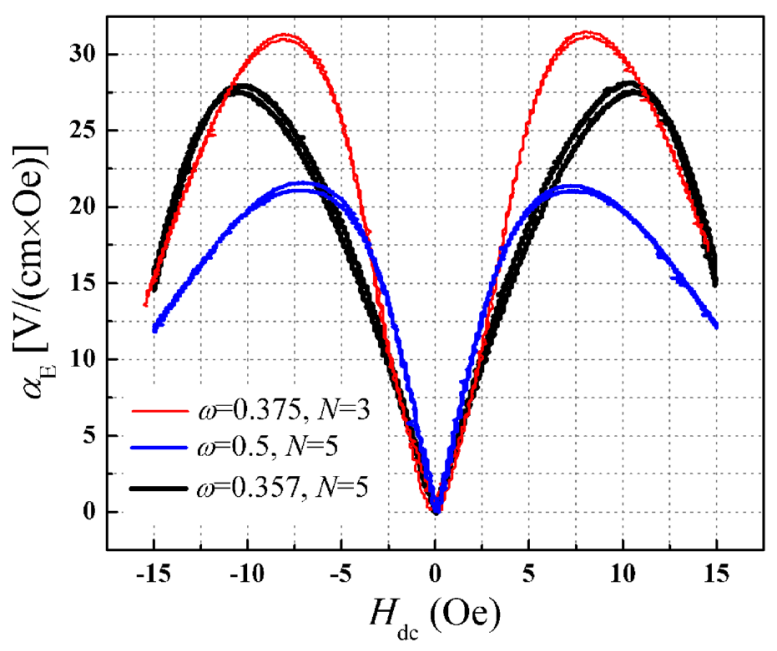

FIG. 3. Measured ME coefficient $\alpha_{\mathrm{E}}$ as a function of dc magnetic bias $H_{\mathrm{dc}}$ for various piezofiber width fraction $\omega$.
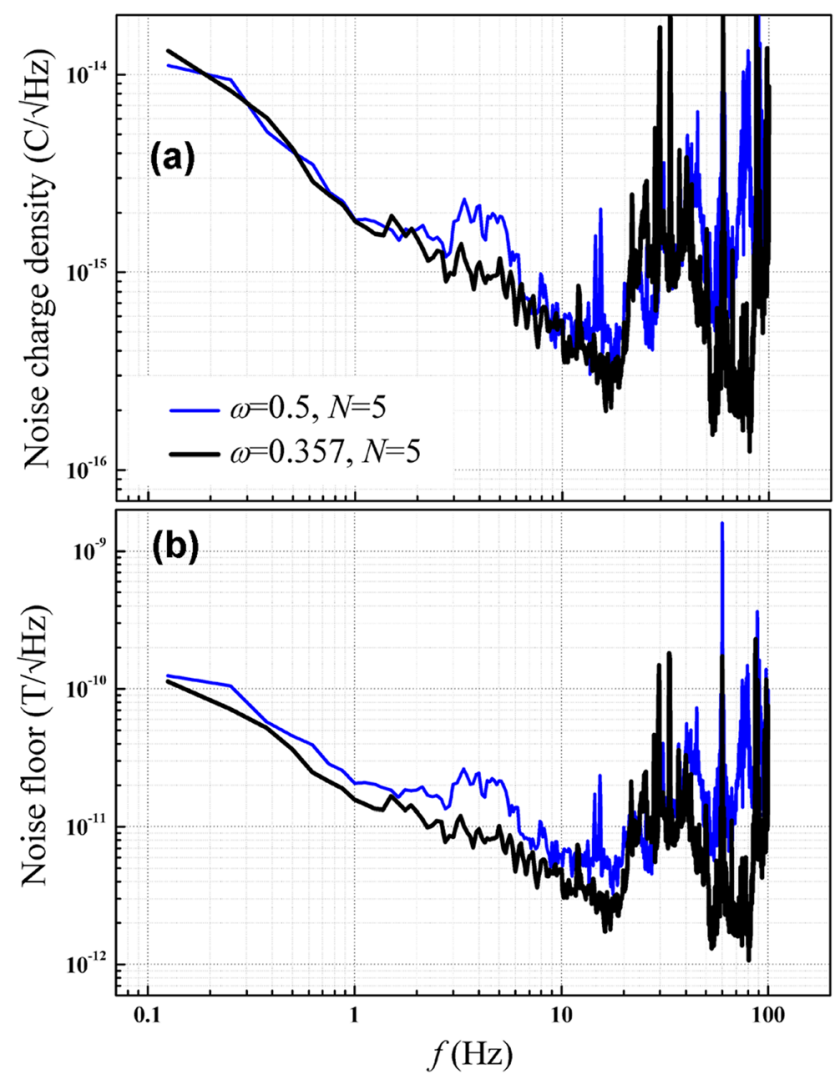

FIG. 4. Noise charge densities and equivalent magnetic noises for sensor units with $\omega=0.357$ and 0.5 .

indicates that there remains notable potential to further enhance the values of $\alpha_{\mathrm{E}}$ by improving the interfacial bonding conditions. ${ }^{11}$ Additionally, we can see the values of $\alpha_{\mathrm{E}}$ for $\omega=0.357$ was even lower than that for $\omega=0.375$. This might be due to higher demagnetization effects in Metglas when wider layers are used. ${ }^{12}$

After measuring the ME properties, the two Metglas/ PZT-fiber heterostructures with piezofiber width fractions of $\omega=0.357$ and 0.5 were assembled in a plastic box with a pair of permanent magnets and a low noise charge amplifier to form sensor units. More details on sensor unit assembly and noise floor measurements can be found in previous publications. ${ }^{6,10}$ Figures 4(a) and 4(b) show the measured noise charge densities and equivalent magnetic noises of the two sensor units in the frequency range of $0.125 \mathrm{~Hz}<f<100 \mathrm{~Hz}$. It can be seen that the noise charge density of the two sensor units exhibited similar trends and magnitudes. This is because the noise sources of the two sensor units were the same: ${ }^{6}$ i.e., capacitance $C=406$ and $397 \mathrm{pF}, \tan \delta=0.0125$ and 0.0123 , dc resistance $R_{\mathrm{dc}}=55$ and $60 \mathrm{G} \Omega$. Nevertheless, the equivalent magnetic noise of the sensor with $\omega=0.357$ was lower than that with $\omega=0.5$, over the entire frequency range except where external vibrational noise sources were present. At an interesting frequency of $f=1 \mathrm{~Hz}$, the equivalent magnetic noise of the two sensors were 15.6 and $20.6 \mathrm{pT} / \sqrt{ } \mathrm{Hz}$. This 1.3 times reduction in the equivalent magnetic noise obviously originates from the geometry-induced ME enhancement.

In summary, an increase in the piezofiber layer width fraction has been shown to result in an enhancement in the ME coefficient and a reduction in the equivalent magnetic 
noise for Metglas/PZT based heterostructures. It was theoretically and experimentally found that a structure with piezofiber width fractions of 0.357 or 0.375 had $1.3-1.5$ times higher ME coefficients, and 1.3 times lower equivalent magnetic noises than that with Metglas width fractions of 0.5 .

This work was sponsored by the Office of Naval Research.

${ }^{1}$ H. Zheng, J. Wang, S. E. Lofland, Z. Ma, L. Mohaddes-Ardabili, T. Zhao, L. Salamanca-Riba, S. R. Shinde, S. B. Ogale, F. Bai, D. Viehland, Y. Jia, D. G. Schlom, M. Wuttig, A. Roytburd, and R. Ramesh, Science 303, 661 (2004).

${ }^{2}$ W. Eerenstein, M. Wiora, J. L. Prieto, J. F. Scott, and N. D. Mathur, Nature Mater. 6, 348 (2007).

${ }^{3}$ J. Ma, J. Hu, Z. Li, and C.-W. Nan, Adv. Mater. 23, 1062 (2011).

${ }^{4}$ R. Ramesh and N. A. Spaldin, Nature Mater. 6, 21 (2007).
${ }^{5}$ S. Valencia, A. Crassous, L. Bocher, V. Garcia, X. Moya, R. O. Cherifi, C. Deranlot, K. Bouzehouane, S. Fusil, A. Zobelli, A. Gloter, N. D. Mathur, A. Gaupp, R. Abrudan, F. Radu, A. Barthelemy, and M. Bibes, Nature Mater. 10, 753 (2011)

${ }^{6}$ Y. J. Wang, D. Gray, D. Berry, J. Q. Gao, M. H. Li, J. F. Li, and D. Viehland, Adv. Mater. 23, 4111 (2011).

${ }^{7}$ M. Bichurin, V. Petrov, and G. Srinivasan, Phys. Rev. B 68, 054402 (2003).

${ }^{8}$ D. Hasanyan, Y. J. Wang, J. Q. Gao, M. H. Li, J. F. Li, and D. Viehland, "Modeling of dynamic behavior of ferromagnetic-ferroelectric-substrate multilayer composites: Longitudinal and bending modes" (unpublished).

${ }^{9}$ J. Das, J. Gao, Z. Xing, J. F. Li, and D. Viehland, Appl. Phys. Lett. 95, 092501 (2009).

${ }^{10}$ M. Li, Y. Wang, J. Gao, D. Gray, J. Li, and D. Viehland, J. Appl. Phys. 111, 033923 (2012).

${ }^{11}$ Y. Wang, D. Gray, D. Berry, M. Li, J. Gao, J. Li, and D. Viehland, J. Alloys Compd. 513, 242 (2012).

${ }^{12}$ C.-M. Chang and G. Carman, Phys. Rev. B 76, 134116 (2007). 